\title{
ON THE INFLUENCE OF WALL PROPERTIES IN THE PERISTALTIC MOTION OF MICROPOLAR FLUID
}

\author{
P. MUTHU ${ }^{1}$, B. V. RATHISH KUMAR ${ }^{1}$ and PEEYUSH CHANDRA ${ }^{1}$
}

(Received 21 June, 2000; revised 6 September, 2001)

\begin{abstract}
We carry out a study of the peristaltic motion of an incompressible micropolar fluid in a two-dimensional channel. The effects of viscoelastic wall properties and micropolar fluid parameters on the flow are investigated using the equations of the fluid as well as of the deformable boundaries. A perturbation technique is used to determine flow characteristics. The velocity profile is presented and discussed briefly. We find the critical values of the parameters involving wall characteristics, which cause mean flow reversal.
\end{abstract}

\section{Introduction}

Expansion and contraction of an extensible tube in a fluid generate progressive waves which propagate along the length of the tube, mixing and transporting the fluid in the direction of wave propagation. This phenomenon is known as peristalsis. It is an inherent property of many tubular organs of the human body. In some biomedical instruments, such as heart-lung machines, peristaltic motion is used to pump blood and other biological fluids. It plays an indispensable role in transporting many physiological fluids in the body in various situations such as (i) urine transport from the kidney to the bladder through the ureter, (ii) transport of spermatozoa in the ductus efferentes of the male reproductive tract, (iii) movement of ovum in the fallopian tubes, (iv) vasomotion of small blood vessels, (v) mixing and transporting the contents of the gastrointestinal passage, and so forth. Peristaltic pumping mechanisms have been utilised for the transport of slurries, sensitive or corrosive fluids, sanitary fluid, noxious fluids in the nuclear industry, to name but a few examples. In some cases the transport of fluids is possible without moving internal mechanical components as is the case with peristaltically operated microelectromechanical system devices [18].

\footnotetext{
${ }^{1}$ Deparment of Mathematics, Indian Institute of Technology, Kanpur 208 016, India;

e-mail: peeyush@iitk.ac.in.

(C) Australian Mathematical Society 2003, Serial-fee code 1446-1811/03
} 
The study of peristalsis in the context of fluid mechanics has received considerable attention in the last three decades mainly because of its relevance to biological systems and industrial applications. Several studies have been made analysing both theoretical and experimental aspects of the peristaltic motion of a Newtonian fluid [5, 8, 19, 24]. Numerical studies have also been reported in the literature $[4,17,22,23]$. In these cases, the relevant fluid is assumed to be Newtonian. For a more detailed understanding of peristaltic transport, we refer to the review articles by Jaffrin and Shapiro [10] and Srivastava and Srivastava [21].

It is well-known that many physiological fluids behave in general like suspensions of deformable or rigid particles in a Newtonian fluid. Blood, for example, is a suspension of red cells, white cells and platelets in plasma. Another example is cervical mucus, which is a suspension of macromolecules in a water-like liquid. In view of this, some researchers have tried to account for the suspension behaviour of biofluids by considering them to be non-Newtonian $[3,15,16,20]$.

Eringen [7] introduced the concept of simple microfluids to characterise concentrated suspensions of neutrally buoyant deformable particles in a viscous fluid where the individuality of substructures affects the physical outcome of the flow. Such fluid models can be used to rheologically describe polymeric suspensions, normal human blood etceter $a$ and have found applications in physiological and engineering problems $[1,2,13]$. A subclass of these microfluids is known as micropolar fluids where the fluid microelements are considered to be rigid [6]. Basically, these fluids can support couple stresses and body couples and exhibit microrotational and microinertial effects. The main advantage of using a micropolar fluid model to study the peristaltic flow of suspensions in comparison with other classes of non-Newtonian fluids is that it takes care of the rotation of fluid particles by means of an independent kinematic vector called the microrotation vector.

Girija Devi and Devanathan [9] studied the peristaltic motion of a micropolar fluid in a cylindrical tube with a sinusoidal wave of small amplitude travelling down its flexible wall for the case of low Reynolds number flow devoid of wall properties like tension and damping. However, consideration of wall properties is essential in various real situations. Mittra and Prasad [12] analysed the peristaltic motion of Newtonian fluid by considering the influence of the viscoelastic behaviour of walls. They assumed that the driving mechanism is in the form of a sinusoidal wave of moderate amplitude imposed on the flexible walls of the channel. Dynamic boundary conditions were proposed for the fluid motion due to the symmetric motion of the flexible walls which were assumed to be either thin elastic plates or membranes [12]. The present study attempts to understand the influence of sinusoidally varying walls on the peristaltic motion of micropolar fluid in a channel using the dynamic boundary condition. Using perturbation techniques, analytical approximate solutions for the stream function and microrotation velocity have been obtained as a power series in terms of the small 
amplitude ratio. Results are discussed for various parameters of the flow and are depicted graphically.

\section{Mathematical model and the governing equations}

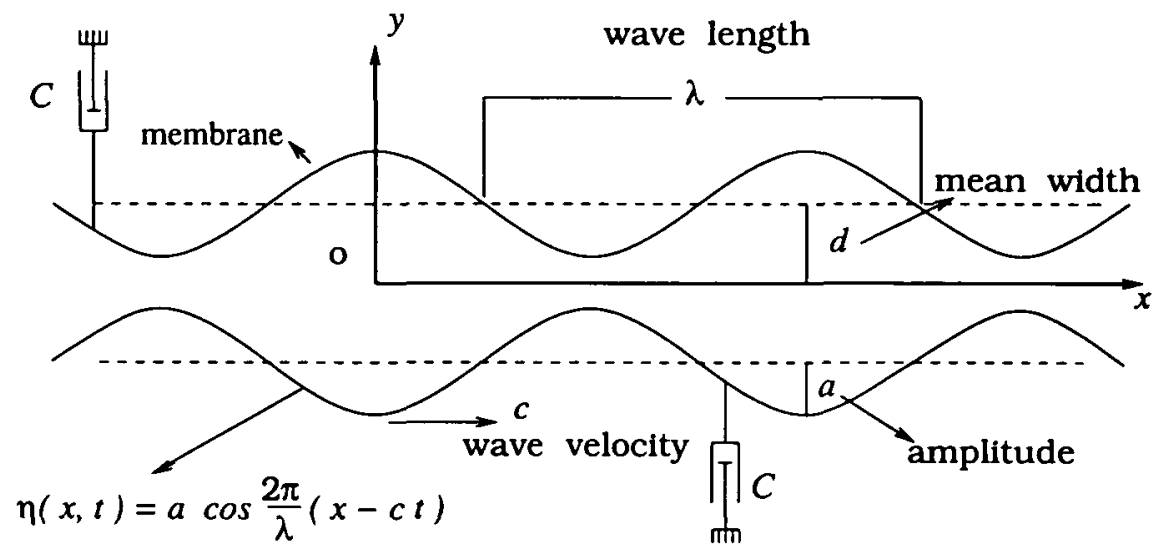

FIGURE 1. Geometry of a two-dimensional peristaltic channel

Consider a two-dimensional symmetric flow of unsteady incompressible micropolar fluid in an infinite channel of uniform thickness $2 d$, with a sinusoidal wave travelling along the walls of the channel with speed $c$, small amplitude $a$ and long wave length $\lambda$ (see Figure 1). The walls are assumed to be flexible membranes. The governing equations for the peristaltic motion of an incompressible micropolar fluid are given in Cartesian form (by neglecting the body forces and body couples) [14] as

$$
\begin{aligned}
\frac{\partial u}{\partial x}+\frac{\partial v}{\partial y} & =0 \\
\rho\left[\frac{\partial u}{\partial t}+u \frac{\partial u}{\partial x}+v \frac{\partial u}{\partial y}\right] & =-\frac{\partial p}{\partial x}+\left(\frac{2 \mu+\kappa}{2}\right) \nabla^{2} u+\kappa \frac{\partial v}{\partial y}, \\
\rho\left[\frac{\partial v}{\partial t}+u \frac{\partial v}{\partial x}+v \frac{\partial v}{\partial y}\right] & =-\frac{\partial p}{\partial y}+\left(\frac{2 \mu+\kappa}{2}\right) \nabla^{2} v-\kappa \frac{\partial v}{\partial x} \\
\rho J\left[\frac{\partial v}{\partial t}+u \frac{\partial v}{\partial x}+v \frac{\partial \nu}{\partial y}\right] & =-2 \kappa \nu+\gamma \nabla^{2} v+\kappa\left[\frac{\partial v}{\partial x}-\frac{\partial u}{\partial y}\right],
\end{aligned}
$$

where $u(x, y, t)$ and $v(x, y, t)$ are the velocity components in the $x$ and $y$ directions respectively, $v(x, y, t)$ is the microrotation velocity component in the direction normal to both the $x$ and $y$ axes and the origin is taken at the centre line of the channel (Figure 1). Here $J$ is the microinertia constant, $\mu$ is the viscosity coefficient of 
classical fluid dynamics, $\kappa$ and $\gamma$ are the new viscosity coefficients for the micropolar fluids, $\rho$ is the density of the fluid and $\nabla^{2} \equiv \partial^{2} / \partial x^{2}+\partial^{2} / \partial y^{2}$.

The displacement $(\eta(x, t))$ of the wall of the channel is given by

$$
\eta(x, t)=a \cos (2 \pi(x-c t) / \lambda)
$$

We assume that the walls are inextensible so that only lateral motion takes place and the horizontal displacement of the wall is zero.

Thus the no-slip boundary conditions for the velocity and microrotation are

$$
u=0, \quad v=0
$$

at $y= \pm(d+\eta(x, t))$.

It may be mentioned that the main purpose of the present study is to understand the dynamic interaction of the fluid and the walls in peristalsis. So the dynamic boundary conditions are imposed on the fluid by the symmetric motion of the flexible walls, which, following Mittra and Prasad [12], can be written as

$$
\frac{\partial L(\eta)}{\partial x}=-\rho\left[\frac{\partial u}{\partial t}+u \frac{\partial u}{\partial x}+v \frac{\partial u}{\partial y}\right]+\left(\frac{2 \mu+\kappa}{2}\right) \nabla^{2} u+\kappa \frac{\partial v}{\partial y}
$$

at $y= \pm(d+\eta(x, t))$, where

$$
\frac{\partial L(\eta)}{\partial x}=-T \frac{\partial^{3} \eta}{\partial x^{3}}+m \frac{\partial^{3} \eta}{\partial t^{2} \partial x}+C \frac{\partial^{2} \eta}{\partial t \partial x} .
$$

Here $T$ is the tension in the membrane, $m$ is the mass per unit area and $C$ is the coefficient of viscous damping force.

Introducing the stream function, $\Psi$, in terms of

$$
u=\frac{\partial \Psi}{\partial y}, \quad v=-\frac{\partial \Psi}{\partial x}
$$

and eliminating the pressure between (2.2) and (2.3), we get differential equations for $\Psi$ and $\nu$. Using $d$ and $c$ as the characteristic length and characteristic velocity, these equations are non-dimensionalised by introducing the following non-dimensional variables:

$$
\begin{array}{rlrlrl}
x^{\prime} & =\frac{x}{d}, & y^{\prime}=\frac{y}{d}, & t^{\prime}=\frac{t c}{d}, & u^{\prime}=\frac{u}{c}, & v^{\prime}=\frac{v}{c}, \\
v^{\prime}=\frac{v d}{c}, & p^{\prime}=\frac{p}{\rho c^{2}}, & \eta^{\prime}=\frac{\eta}{d}, & \Psi^{\prime}=\frac{\Psi}{c d}, & J^{\prime}=\frac{J}{d^{2}} .
\end{array}
$$


After non-dimensionalisation, the accents are dropped and the governing equations and boundary conditions, in non-dimensional form, are written as

$$
\begin{aligned}
\frac{\partial}{\partial t} \nabla^{2} \Psi+\Psi_{y} \nabla^{2} \Psi_{x}-\Psi_{x} \nabla^{2} \Psi_{y} & =\frac{2+\mu_{1}}{2 R_{e}}\left[\nabla^{2} \nabla^{2} \Psi\right]+\frac{\mu_{1}}{R_{e}} \nabla^{2} \nu \\
R_{l}\left(\frac{\partial \nu}{\partial t}+\Psi_{y} \frac{\partial \nu}{\partial x}-\Psi_{x} \frac{\partial \nu}{\partial y}\right) & =2\left(1-N^{2}\right)\left[\nabla^{2} \nu\right]-N^{2} M^{2}\left[\nabla^{2} \Psi+2 \nu\right]
\end{aligned}
$$

The boundary conditions at $y= \pm(1+\eta(x, t))$ are

$$
\begin{gathered}
\Psi_{y}=0, \quad \nu=0 \\
\frac{\partial L(\eta)}{\partial x}=-\left[\Psi_{y t}+\Psi_{y} \Psi_{x y}-\Psi_{x} \Psi_{y y}\right]+\frac{2+\mu_{1}}{2 R_{e}}\left[\nabla^{2} \Psi_{y}\right]+\frac{\mu_{1}}{R_{e}} \frac{\partial \nu}{\partial y}
\end{gathered}
$$

where

$$
\frac{\partial L(\eta)}{\partial x}=-\frac{K_{3}}{R_{e}^{2}} \frac{\partial^{3} \eta}{\partial x^{3}}+m_{1} \frac{\partial^{3} \eta}{\partial t^{2} \partial x}+\frac{K_{2}}{R_{e}} \frac{\partial^{2} \eta}{\partial t \partial x},
$$

$\eta(x, t)=\epsilon \cos (\alpha(x-t)), \epsilon=a / d, \alpha=2 \pi d / \lambda, R_{e}=\rho c d / \mu, \mu_{1}=\kappa / \mu$ and

$$
\begin{aligned}
N & =\left(\frac{\mu_{1}}{2+\mu_{1}}\right)^{1 / 2}, & M & =2 d\left(\frac{\mu}{\gamma}\right)^{1 / 2}, \\
K_{3} & =\frac{T \rho d}{\mu^{2}}, & K_{2} & =\frac{C d}{\mu}, \\
m_{1} & =\frac{m}{\rho d}, & R_{l} & =\frac{8 \mu \rho c d J}{\gamma(2 \mu+\kappa)} .
\end{aligned}
$$

The parameters $\epsilon, \alpha$ and $R_{e}$ are the amplitude ratio, wave number and Reynolds number respectively. These are the usual fundamental quantities observed in classical peristaltic flow $[8,12]$. The parameters $\mu_{1}$ and $M$ are non-dimensional quantities due to micropolar fluid flow. Also $\mu_{1}$ denotes the ratio of the viscosity coefficient for micropolar fluids and the classical viscosity coefficient. It characterises the coupling of (2.7) and (2.8). The parameter $M$ can be thought of as a fluid property depending upon the size of the microstructure. This is due to the factor $(\gamma / \mu)^{1 / 2}$, which has the dimension of length. It can be noted that as $\kappa$ tends to zero, $\mu_{1}$ becomes zero and (2.7) and (2.8) are uncoupled. Further, when $\kappa$ and $\gamma$ are zero, that is, when $\mu_{1}$ becomes zero and $M$ tends to infinity, (2.7) and (2.8) reduce to the classical Navier-Stokes equations. We note that $R_{l}$ is the modified Reynolds number and involves the quantity $J$ (microinertia constant), where $J$ is the square of a length typical of microstructure, and it is reasonable to assume that $R_{l} \ll 1[11,14]$. In view of this, the effect of microinertia is neglected and $R_{l}$ is taken to be zero in the following analysis.

The parameters $K_{2}, K_{3}$ and $m_{1}$ are the non-dimensional quantities related to the wall motion through the dynamic boundary condition (2.9). The parameters $K_{2}$ and $K_{3}$ respectively represent the dissipative and rigiditive feature of walls, whereas 
$m_{1}$ indicates the stiffness property of walls. The choice $K_{2}=0$ implies that the walls move up and down with no damping force on them and hence indicates the case of elastic walls. The rigid nature of the walls is represented by $K_{3}$, which depends upon the wall tension.

\section{Analysis}

It may be noted that the flow is quite complex because of nonlinearity and the coupled behaviour of the governing equations and the boundary conditions. Thus to solve (2.7) and (2.8) for the velocity field and microrotation, we attempt an approximate solution as a power series in terms of $\epsilon$ (ratio of amplitude to mean breadth of the wavy wall). The pressure gradient is taken as

$$
\frac{\partial p}{\partial x}=\left(\frac{\partial p}{\partial x}\right)_{0}+\epsilon\left(\frac{\partial p}{\partial x}\right)_{1}+\epsilon^{2}\left(\frac{\partial p}{\partial x}\right)_{2}+\cdots
$$

Further, it may be pointed out here that although the peristaltic motion is caused by a single harmonic wave, the velocity field will consist of all harmonics due to the nonlinearity of the equations. Thus we assume $\Psi$ and $\nu$ are given in the following form:

$$
\begin{aligned}
\Psi= & \Psi_{0}(y)+\frac{\epsilon}{2}\left(\phi_{1}(y) e^{i \alpha(x-t)}+\phi_{1}^{*}(y) e^{-i \alpha(x-t)}\right) \\
& +\frac{\epsilon^{2}}{2}\left(\phi_{20}(y)+\phi_{22}(y) e^{2 i \alpha(x-t)}+\phi_{22}^{\star}(y) e^{-2 i \alpha(x-t)}\right)+o\left(\epsilon^{3}\right), \\
\nu= & \nu_{0}(y)+\frac{\epsilon}{2}\left(\xi_{1}(y) e^{i \alpha(x-t)}+\xi_{1}^{*}(y) e^{-i \alpha(x-t)}\right) \\
& +\frac{\epsilon^{2}}{2}\left(\xi_{20}(y)+\xi_{22}(y) e^{2 i \alpha(x-t)}+\xi_{22}^{*}(y) e^{-2 i \alpha(x-t)}\right)+o\left(\epsilon^{3}\right) .
\end{aligned}
$$

Here the asterisk denotes complex conjugate and $\Psi_{0}(y)$ and $\nu_{0}(y)$ correspond to the plane Poiseuille flow, where $\epsilon=0$. Substituting (3.1) and (3.2) in (2.7) to (2.9) and collecting the terms of various powers of $\epsilon$ and also different harmonics, we get the differential equations for different orders of $\Psi$ and $\nu$ (Appendix A).

Since zeroth-order differential equations correspond to plane Poiseuille flow of micropolar fluid, where $\epsilon=0$, the equations can be easily solved along with the corresponding boundary conditions for $\Psi_{0}$ and $v_{0}$ with a constant pressure gradient $(d p / d x)_{0}$. Thus we get

$$
\begin{aligned}
\frac{d \Psi_{0}}{d y} & =K\left(y^{2}-1\right)+2 K \frac{N}{M}\left(\frac{\cosh (N M)-\cosh (N M y)}{\sinh (N M)}\right), \\
\nu_{0} & =K\left(\frac{\sinh (N M y)-y \sinh (N M)}{\sinh (N M)}\right),
\end{aligned}
$$


where $K=\left(R_{e} /\left(2+\mu_{1}\right)\right)(d p / d x)_{0}$ is the Poiseuille flow parameter for the micropolar fluid.

For pure peristalsis (free pumping), which means that the flow is generated by wall motions only, the pressure gradient $(\partial p / \partial x)_{0}=0$. This implies that $K=0$, which gives $\Psi_{0}(y) \equiv 0, \nu_{0}(y) \equiv 0$.

The time mean flow $\bar{u}(y)$ is defined as the velocity averaged over the period of oscillation $(\tau)$ of the wave propagation imposed on the flexible walls,

$$
\bar{u}(y)=\frac{1}{\tau} \int_{t=0}^{\tau} \frac{\partial \Psi}{\partial y} d t=\Psi_{0}^{(1)}(y)+\frac{\epsilon^{2}}{2} \phi_{20}^{(1)}(y)+o\left(\epsilon^{3}\right) .
$$

Here $f^{(2)}$ means $d^{2} f / d y^{2}$. In the following we shall discuss the case of free pumping only. In that case, we have

$$
\bar{u}(y)=\frac{\epsilon^{2}}{2} \phi_{20}^{(1)}(y)+o\left(\epsilon^{3}\right) .
$$

It is clear from the equations corresponding to the order of $\epsilon^{2}$ that to calculate $\phi_{20}$ for the free pumping case, we need the expression for $\phi_{1}$ and $\xi_{1}$. The governing equations for $\phi_{1}$ and $\xi_{1}$ for the free pumping case reduce to

$$
\begin{array}{r}
\left(\phi_{1}^{(4)}-2 \alpha^{2} \phi_{1}^{(2)}+\alpha^{4} \phi_{1}\right)+2 \mu_{1}\left(\xi_{1}^{(2)}-\alpha^{2} \xi_{1}\right)=\frac{2 i \alpha R_{e}}{2+\mu_{1}}\left(\alpha^{2} \phi_{1}-\phi_{1}^{(2)}\right), \\
2\left(1-N^{2}\right)\left(\xi_{1}^{(2)}-\alpha^{2} \xi_{1}\right)-2 N^{2} M^{2} \xi_{1}-N^{2} M^{2}\left(\phi_{1}^{(2)}-\alpha^{2} \phi_{1}\right)=0
\end{array}
$$

and the boundary conditions are

$$
\begin{gathered}
\phi_{1}^{(1)}( \pm 1)=0, \quad \xi_{1}( \pm 1)=0 \\
\phi_{1}^{(3)}( \pm 1)+2 \mu_{1} \xi_{1}^{(1)}( \pm 1)=\frac{2 R_{e} \delta_{1}}{2+\mu_{1}}
\end{gathered}
$$

where $\delta_{1}=i\left(K_{3} \alpha^{3} / R_{e}^{2}-m_{1} \alpha^{3}\right)+K_{2} \alpha^{2} / R_{e}$.

It is clear from (3.3) that to determine $\bar{u}(y)$ up to the second order of $\epsilon$, we need the expression for $\phi_{20}$. Thus in the following we give the equations governing $\phi_{20}$ only, for the case of free pumping,

$$
\begin{gathered}
\phi_{20}^{(4)}+2 \mu_{1} \xi_{20}^{(2)}=\frac{-i \alpha R_{e}}{\left(2+\mu_{1}\right)}\left(\phi_{1} \phi_{1}^{*(2)}-\phi_{1}^{*} \phi_{1}^{(2)}\right)^{(1)}, \\
2\left(1-N^{2}\right) \xi_{20}^{(2)}-2 N^{2} M^{2} \xi_{20}-N^{2} M^{2} \phi_{20}^{(2)}=0,
\end{gathered}
$$

with boundary conditions

$$
\phi_{20}^{(1)}( \pm 1)=\mp \frac{1}{2}\left(\phi_{1}^{(2)}( \pm 1)+\phi_{1}^{*(2)}( \pm 1)\right), \quad \xi_{20}( \pm 1)=\mp \frac{1}{2}\left(\xi_{1}^{(1)}( \pm 1)+\xi_{1}^{*(1)}( \pm 1)\right),
$$


and

$$
\begin{aligned}
\phi_{20}^{(3)}( \pm 1) & +2 \mu_{1} \xi_{20}^{(1)}( \pm 1) \\
= & \pm \frac{i \alpha R_{e}}{\left(2+\mu_{1}\right)}\left(\phi_{1}^{*(2)}( \pm 1)-\phi_{1}^{(2)}( \pm 1)\right) \pm \frac{\alpha^{2}}{2}\left(\phi_{1}^{(2)}( \pm 1)+\phi_{1}^{*(2)}( \pm 1)\right) \\
& \mp \frac{1}{2}\left(\phi_{1}^{(4)}( \pm 1)+\phi_{1}^{*(4)}( \pm 1)\right) \\
& -\frac{i \alpha R_{e}}{\left(2+\mu_{1}\right)}\left(\phi_{1}( \pm 1) \phi_{1}^{*(2)}( \pm 1)-\phi_{1}^{*}( \pm 1) \phi_{1}^{(2)}( \pm 1)\right) \\
& \mp N^{2}\left(\xi_{1}^{(2)}( \pm 1)+\xi_{1}^{*(2)}( \pm 1)\right) .
\end{aligned}
$$

Solutions for $\phi_{1}(y), \xi_{1}(y)$ and $\phi_{20}(y)$ are given in Appendix B and $\bar{u}(y)$ is calculated from (3.3).

\section{Numerical results and discussion}

In the following we study the effects of various parameters on the time mean flow for the case of pure peristalsis only. The time mean flow, $\bar{u}(y)$, approximated up to order $\epsilon^{2}$, is given by (3.3). It may be noted that, apart from the usual dimensionless parameters of peristaltic flow, $R_{e}$ (Reynolds number), $\alpha$ (wave number) and $\epsilon$ (amplitude ratio), in this case the flow also depends upon the following non-dimensional quantities:

(1) the micropolar fluid parameters $\mu_{1}$ and $M$; and

(2) the wall parameters $K_{2}, K_{3}$ and $m_{1}$, which describe the viscoelastic behaviour of the flexible walls.

We mention here that $\mu_{1}$ and $M$ characterise the coefficient of viscosity $(\kappa)$ and of gyroviscosity $(\gamma)$ respectively. An increase in $\kappa$ is reflected in the coupling parameter $\mu_{1}$, while an increase in $\gamma$ results in decreasing values of $M$. Also the expression for $\bar{u}(y)$ reduces to the Newtonian case [12], as $\mu_{1} \rightarrow 0$ and $M \rightarrow \infty$.

The parameters $K_{2}$ and $K_{3}$ represent respectively the dissipative and rigiditive features of the walls while $m_{1}$ indicates the stiffness property of the walls. The choice $K_{2}=0$ implies that the walls move up and down with no damping force on them and hence indicates the case of elastic walls. The rigid nature of the walls is represented by $\dot{K_{3}}$. In our analysis we assume that there is no stiffness in the walls and this implies $m_{1}=0$. It may be noted from (2.5) that these three quantities cannot be taken as zero simultaneously.

We shall now consider the following two cases for the wall properties:

(i) elastic wall $\left(K_{2}=0\right)$;

(ii) dissipative wall $\left(K_{2} \neq 0\right)$. 


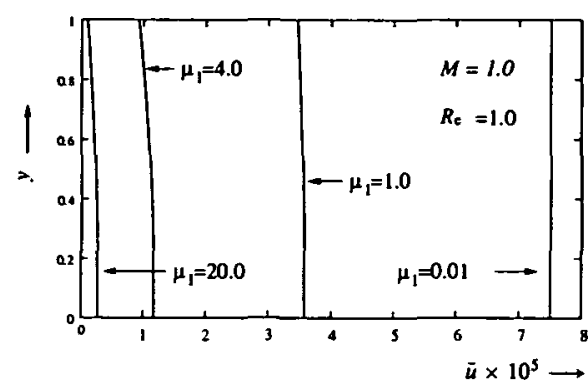

(a)

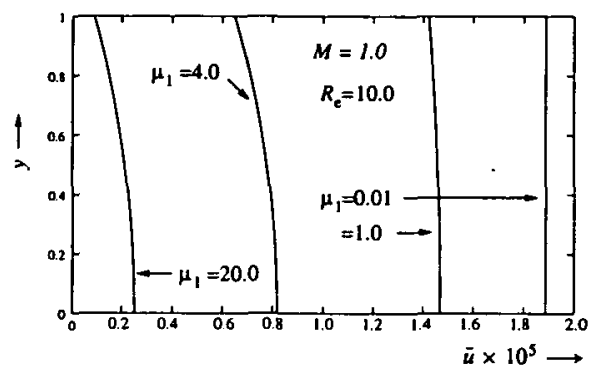

(c)

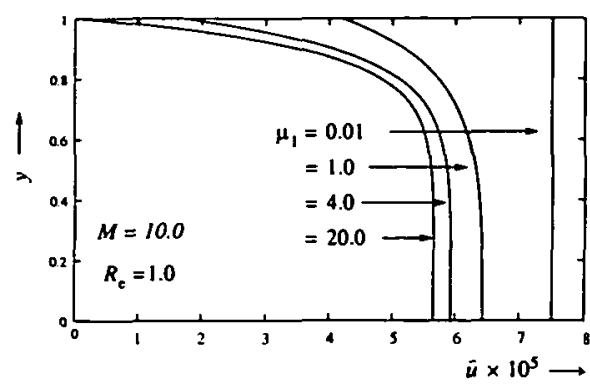

(b)

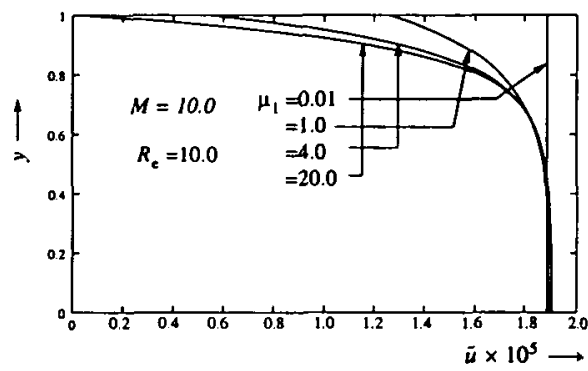

(d)

FIGURE 2. Distribution of $\bar{u}$ with $y$ for $\alpha=0.5\left(K_{2}=0, K_{3}=1.0, m_{1}=0\right)$

Case (i): $K_{2}=0$. This corresponds to elastic walls where the dissipative effects are neglected. In this case we numerically study the effects of fluid parameters and $\alpha$ (wave number) on the mean flow characteristics, by taking $K_{3}=1.0$ and $m_{1}=0.0$ in a channel where $\epsilon=0.1$.

Figures 2 and 3 show the effect of $\mu_{1}, M$ and $R_{e}$ on the time mean flow, induced by the travelling waves on the flexible walls, when $\alpha=0.5$ and $\alpha=2.0$ respectively.

The effect of $\gamma$ (the coefficient of gyroviscosity) is observed by comparing the graphs for $M=1$ (Figures 2 (a), (c) and 3 (a), (c)) and for $M=10$ (Figures 2 (b), (d) and $3(\mathrm{~b}),(\mathrm{d}))$. It is seen here that $\bar{u}(y)$ is large for higher values of $M(M=10)$. Further, when $M=1$, the flow is almost like a plug flow and $\bar{u}(y)$ is almost constant. However, for $M=10$, the plug flow is observed in most parts of the channel and $\bar{u}(y){ }^{\circ}$ sharply decreases to zero near the walls. In the case where $M=10$, the variation with respect to $\mu_{1}$ is not very significant.

To see the influence of the Reynolds number $\left(R_{e}\right)$, we have considered the two cases $R_{e}=1$ and $R_{e}=10$. It is seen that $\bar{u}(y)$ decreases as $R_{e}$ increases. Further, it is observed that while the qualitative behaviour of $\bar{u}(y)$ with respect to $\mu_{1}$ and $M$ remains similar in the two cases for $R_{e}$, the effect of $\mu_{1}$ is more significant at low Reynolds number, when $M=10$. 


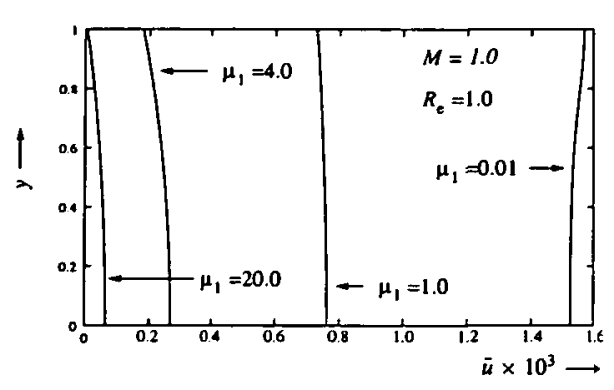

(a)

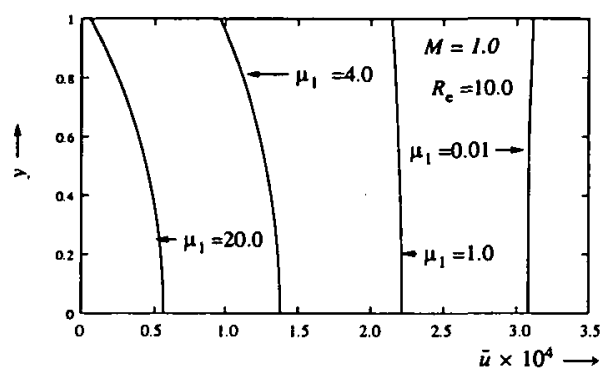

(c)

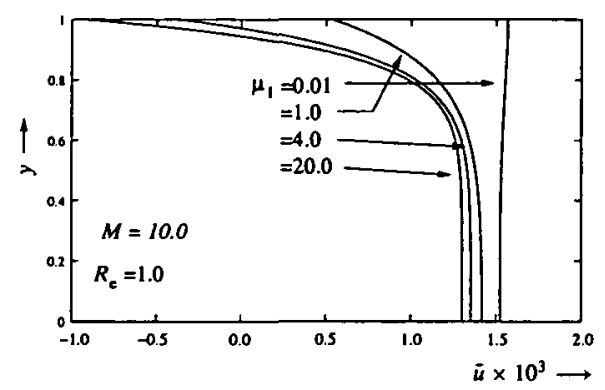

(b)

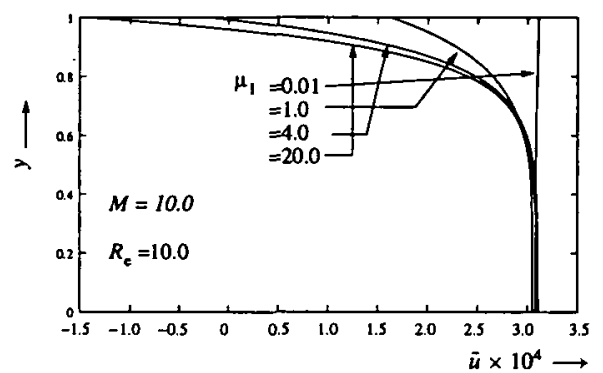

(d)

FIGURE 3. Distribution of $\bar{u}$ with $y$ for $\alpha=2.0\left(K_{2}=0, K_{3}=1.0, m_{1}=0\right)$

The same analysis is repeated with $\alpha=2.0$. The pictorial representation for this analysis is given in Figure 3. Comparing Figures 2 and 3, it is observed that $\vec{u}(y)$ increases with an increase in $\alpha$.

In Figure 4, we see the effect of $K_{3}$ on $\bar{u}(y)$ for the case of pure peristalsis with elastic walls $\left(K_{2}=0\right.$ ), by taking $\alpha=1, R_{e}=10$ and $m_{1}=0$. It is observed that as $K_{3}$ increases, $\bar{u}(1)$ increases and $\bar{u}(0)$ decreases. In fact $\bar{u}(y)$ remains positive throughout the channel until $K_{3}$ attains a critical value $K_{3 c}$, for which $\bar{u}(0)=0$, and after that it starts showing flow reversal behaviour at the middle of the channel, that is, when $y=0$. It is seen from Figure 4 that the value of $K_{3 c}$ depends upon $\mu_{1}$ and $M$. It increases as $\mu_{1}$ increases and decreases with $M$. As larger values of $K_{3}$ correspond to higher rigidity of the flexible boundary walls, one can conclude that higher rigidity of the walls is required for mean flow reversal to take place in the case of micropolar fluids.

Case (ii): Dissipative wall $\left(K_{2} \neq 0\right.$ ). In the next two figures (5 and 6), we see the effect of the dissipative nature of the walls $\left(K_{2} \neq 0\right)$ on the flow reversal by taking $K_{3}=1$ and $m_{1}=0$. To see this effect, $\bar{u}(1)$, that is, time mean flow at the boundary is plotted versus $\alpha$ (wave number). While it is observed that no flow reversal takes 


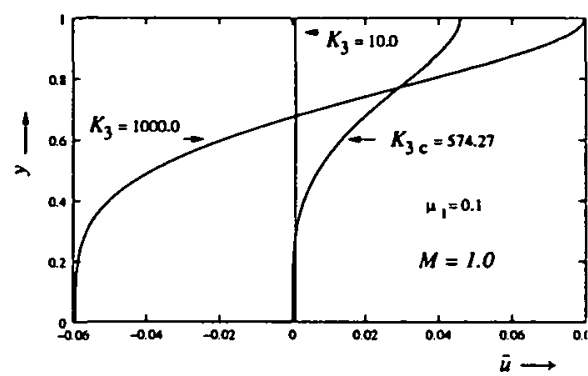

(a)

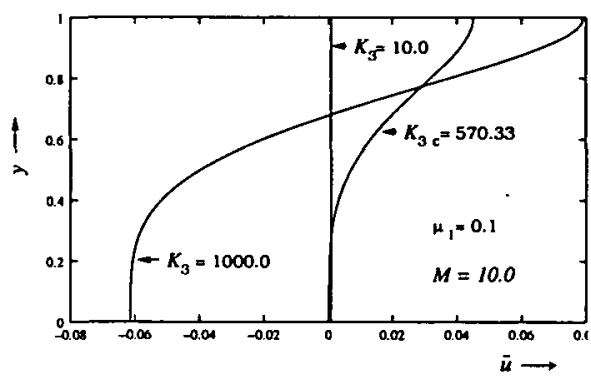

(c)

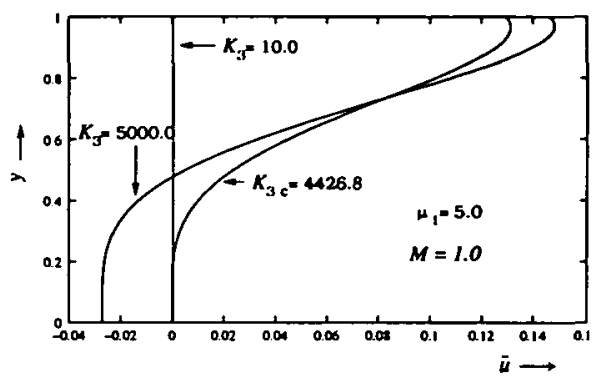

(b)

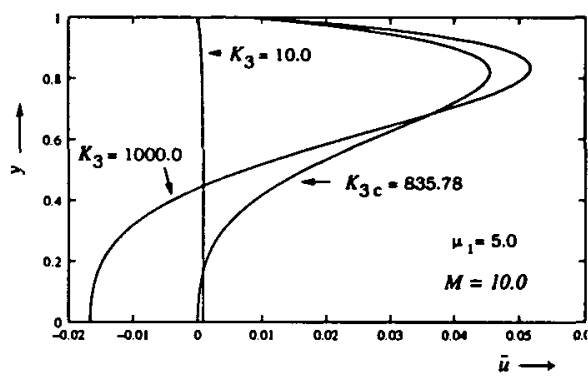

(d)

FIGURE 4. Mean velocity distribution for $\alpha=1.0, R_{e}=10.0\left(K_{2}=0, m_{1}=0\right)$

place at the boundary for smaller values of $K_{2}(M=1$, Figure 5), for any value of $\alpha$, $\bar{u}(1)$ is negative for larger values of $K_{2}$ and it keeps on decreasing as $\alpha$ increases.

Thus the dissipative nature of the walls causes flow reversal at the boundary. This is observed for various values of $\mu_{1}, M$ and $R_{e}$. We note that in the case of non-dissipative walls this phenomenon was not observed. The effect of the coupling parameter $\mu_{1}$ is to decrease the values of $\bar{u}(1)$ and the effect of $M$ is not very significant (Figures 5 (a) and 6 (a)). The effect of $R_{e}$ is to decrease the value of $\bar{u}(1)$ at small values of $K_{2}$ and $\alpha$. At large values of $K_{2}$ the reverse effect is observed at all values of $\alpha$, except in the case of $\mu_{1}=10$ and $M=10$ (Figure $6(\mathrm{~b})$ ). This peculiar behaviour of $\bar{u}(1)$ (Figure $6(\mathrm{~b})$ ) is due to the combined effect of $\mu_{1}$ and $R_{e}$ as $\bar{u}(1)$ decreases with $\mu_{1}$ and increases with $R_{e}$ for larger value of $K_{2}$.

\section{Conclusion}

In the present study an analysis of peristaltic transport of a micropolar fluid in a channel with dynamic boundary conditions has been presented. It is observed that $\bar{u}(y)$ decreases when $\mu_{1}$ increases and as $M$ decreases. The phenomenon of flow 


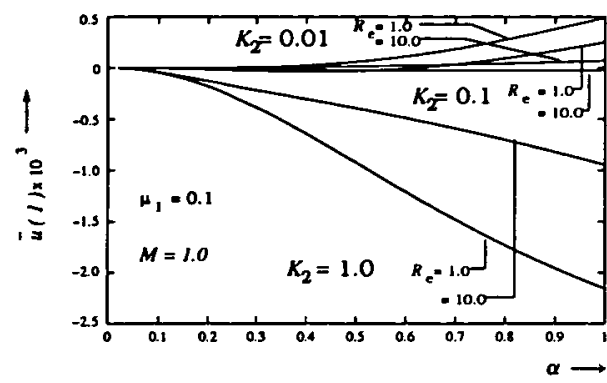

(a)

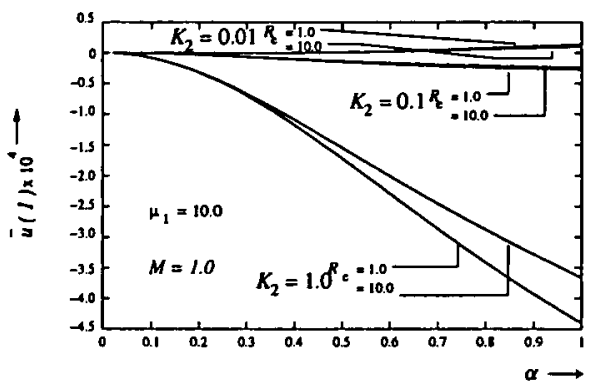

(b)

FIGURE 5. Effect of viscous damping on $\bar{u}(1)\left(K_{3}=1.0, m_{1}=0.0\right)$

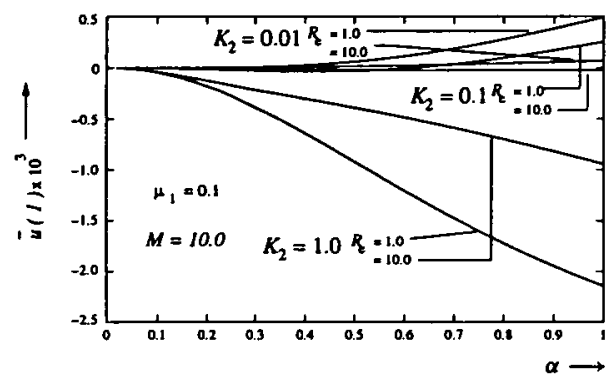

(a)

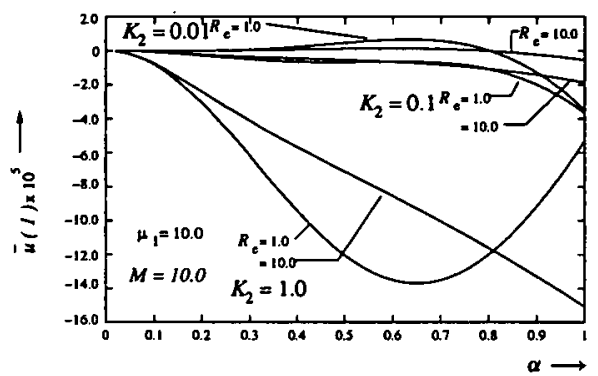

(b)

FIGURE 6. Effect of viscous damping on $\bar{u}(1)\left(K_{3}=1.0, m_{1}=0.0\right)$

reversal takes place in the centerline of the channel due to rigidity variation in the boundary walls, when viscous damping is zero. The critical value of $K_{3}$ increases with an increase in $\mu_{1}$ and it decreases as $M$ increases. For non-zero viscous damping, flow reversal is found at the channel walls. Furthermore, these flow variables approach behaviour in accordance with classical theory as the microstructure loses its behaviour.

Acknowledgement. The authors are thankful to the referees for their constructive remarks.

\section{Appendix A.}

The governing equations for $\Psi_{0}$ and $\nu_{0}$ are

$$
\begin{array}{r}
\left(2+\mu_{1}\right) \Psi_{0}^{(4)}+2 \mu_{1} v_{0}^{(2)}=0, \\
2\left(1-N^{2}\right)\left[v_{0}^{(2)}\right]-N^{2} M^{2}\left[\Psi_{0}^{(2)}+2 v_{0}\right]=0,
\end{array}
$$


with boundary conditions given by $\Psi_{0}^{(1)}( \pm 1)=0, v_{0}( \pm 1)=0$. The governing equations for $\phi_{1}$ and $\xi_{1}$ are

$$
\begin{gathered}
\frac{2+\mu_{1}}{2 R_{e}}\left(\phi_{1}^{(4)}-2 \alpha^{2} \phi_{1}^{(2)}+\alpha^{4} \phi_{1}\right)+\frac{\mu_{1}}{R_{e}}\left(\xi_{1}^{(2)}-\alpha^{2} \xi_{1}\right) \\
=i \alpha\left(1-\Psi_{0}^{(1)}\right)\left(\alpha^{2} \phi_{1}-\phi_{1}^{(2)}\right)-i \alpha \Psi_{0}^{(3)} \phi_{1}, \\
2\left(1-N^{2}\right)\left(\xi_{1}^{(2)}-\alpha^{2} \xi_{1}\right)-2 N^{2} M^{2} \xi_{1}-N^{2} M^{2}\left(\phi_{1}^{(2)}-\alpha^{2} \phi_{1}\right)=0,
\end{gathered}
$$

with boundary conditions given by

$$
\begin{gathered}
\phi_{1}^{(1)}( \pm 1)=\mp \Psi_{0}^{(2)}( \pm 1), \quad \xi_{1}( \pm 1)=\mp v_{0}^{(1)}( \pm 1), \\
\frac{2+\mu_{1}}{2 R_{e}}\left(\phi_{1}^{(3)}( \pm 1)-\alpha^{2} \phi_{1}^{(1)}( \pm 1) \pm \Psi_{0}^{(4)}( \pm 1)\right)+i \alpha \phi_{1}^{(1)}( \pm 1)\left(1-\Psi_{0}^{(1)}( \pm 1)\right) \\
+i \alpha \phi_{1}( \pm 1) \Psi_{0}^{(2)}( \pm 1)+\frac{\mu_{1}}{R_{e}}\left(\xi_{1}^{(1)}( \pm 1) \pm \nu_{0}^{(2)}( \pm 1)\right)=\delta_{1},
\end{gathered}
$$

where $\delta_{1}=i\left(K_{3} \alpha^{3} / R_{e}^{2}-m_{1} \alpha^{3}\right)+K_{2} \alpha^{2} / R_{e}$. The governing equations for $\phi_{20}$ and $\xi_{20}$ are

$$
\begin{gathered}
\frac{2+\mu_{1}}{2 R_{e}} \phi_{20}^{(4)}+\frac{\mu_{1}}{R_{e}} \xi_{20}^{(2)}=\frac{-i \alpha}{2}\left(\phi_{1} \phi_{1}^{*(2)}-\phi_{1}^{*} \phi_{1}^{(2)}\right)^{(1)}, \\
2\left(1-N^{2}\right) \xi_{20}^{(2)}-2 N^{2} M^{2} \xi_{20}-N^{2} M^{2} \phi_{20}^{(2)}=0,
\end{gathered}
$$

with boundary conditions given by

$$
\begin{aligned}
\phi_{20}^{(1)}( \pm 1)= & \mp \frac{1}{2}\left(\phi_{1}^{(2)}( \pm 1)+\phi_{1}^{*(2)}( \pm 1)\right)-\frac{1}{2} \Psi_{0}^{(3)}( \pm 1), \\
\xi_{20}( \pm 1)= & \mp \frac{1}{2}\left(\xi_{1}^{(1)}( \pm 1)+\xi_{1}^{*(1)}( \pm 1)\right)-\frac{1}{2} \nu_{0}^{(2)}( \pm 1), \\
\phi_{20}^{(3)}( \pm 1)+\mu_{1} \xi_{20}^{(1)}( \pm 1)= & \pm \frac{i \alpha R_{e}}{\left(2+\mu_{1}\right)}\left(\phi_{1}^{*(2)}( \pm 1)-\phi_{1}^{(2)}( \pm 1)\right) \\
& \pm \frac{\alpha^{2}}{2}\left(\phi_{1}^{(2)}( \pm 1)+\phi_{1}^{*(2)}( \pm 1)\right) \mp \frac{1}{2}\left(\phi_{1}^{(4)}( \pm 1)+\phi_{1}^{*(4)}( \pm 1)\right) \\
& -\frac{1}{2} \Psi_{0}^{(5)}( \pm 1) \pm \frac{i \alpha R_{e}}{\left(2+\mu_{1}\right)} \Psi_{0}^{(3)}( \pm 1)\left(\phi_{1}^{*}( \pm 1)-\phi_{1}( \pm 1)\right) \\
& -\frac{i \alpha R_{e}}{\left(2+\mu_{1}\right)}\left(\phi_{1}( \pm 1) \phi_{1}{ }^{*(2)}( \pm 1)-\phi_{1}^{*}( \pm 1) \phi_{1}^{(2)}( \pm 1)\right) \\
& \mp N^{2}\left(\xi_{1}^{(2)}( \pm 1)+\xi_{1}^{*(2)}( \pm 1)\right)-N^{2} \nu_{0}^{(2)}( \pm 1) .
\end{aligned}
$$

The governing equations for $\phi_{22}$ and $\xi_{22}$ are

$$
\begin{aligned}
& \frac{2+\mu_{1}}{2 R_{e}}\left(\phi_{22}^{(4)}-8 \alpha^{2} \phi_{22}^{(2)}+16 \alpha^{4} \phi_{22}\right)+\frac{\mu_{1}}{R_{e}}\left(\xi_{22}^{(2)}-4 \alpha^{2} \xi_{22}\right) \\
& \quad=2 i \alpha\left(1-\Psi_{0}^{(1)}\right)\left(4 \alpha^{2} \phi_{22}-\phi_{22}^{(2)}\right)-2 i \alpha \Psi_{0}^{(3)} \phi_{22}+\frac{i \alpha}{2}\left(\phi_{1}^{(1)} \phi_{1}^{(2)}-\phi_{1} \phi_{1}^{(3)}\right),
\end{aligned}
$$




$$
2\left(1-N^{2}\right)\left(\xi_{22}^{(2)}-4 \alpha^{2} \xi_{22}\right)-2 N^{2} M^{2} \xi_{22}-N^{2} M^{2}\left(\phi_{22}^{(2)}-4 \alpha^{2} \phi_{22}\right)=0,
$$

with boundary conditions given by

$$
\begin{gathered}
\phi_{22}^{(1)}( \pm 1)=\mp \phi_{1}^{(2)}( \pm 1) / 2-\Psi_{0}^{(3)}( \pm 1) / 4 \\
\xi_{22}( \pm 1)=\mp \xi_{1}^{(1)}( \pm 1) / 2-v_{0}^{(2)}( \pm 1) / 4 \\
\frac{2+\mu_{1}}{2 R_{e}} \phi_{22}^{(3)}( \pm 1)+\left(2 i \alpha-\frac{2\left(2+\mu_{1}\right) \alpha^{2}}{R_{e}}\right) \phi_{22}^{(1)}( \pm 1) \\
+2 i \alpha \Psi_{0}^{(2)}( \pm 1) \phi_{22}( \pm 1)+\frac{\mu_{1}}{R_{e}} \xi_{22}^{(1)}( \pm 1) \\
= \pm \frac{2+\mu_{1}}{4 R_{e}}\left(\alpha^{2} \phi_{1}^{(2)}( \pm 1)-\phi_{1}^{(4)}( \pm 1)\right)-\frac{\left(2+\mu_{1}\right) \Psi_{0}^{(5)}( \pm 1)}{8 R_{e}} \\
+2 i \alpha\left[\left(\phi_{1}^{(1)}( \pm 1)\right)^{2}-\phi_{1}( \pm 1) \phi_{1}^{(2)}( \pm 1) \mp \phi_{1}^{(2)}( \pm 1)\right] / 4 \\
-\frac{i \alpha}{2} \Psi_{0}^{(3)}( \pm 1) \phi_{1}( \pm 1)-\frac{\mu_{1}}{2 R_{e}}\left(\xi_{1}^{(2)}( \pm 1) \pm \frac{\nu_{0}^{(2)}( \pm 1)}{2}\right)
\end{gathered}
$$

\section{Appendix B.}

The solutions to (3.4)-(3.5) are

$$
\begin{aligned}
& \phi_{1}(y)=A_{3} \sinh \alpha y+A_{4} \sinh \beta y+a_{1} A_{1} \sinh r_{1} y+a_{2} A_{2} \sinh r_{2} y, \\
& \xi_{1}(y)=A_{1} \sinh r_{1} y+A_{2} \sinh r_{2} y,
\end{aligned}
$$

where

$$
\begin{gathered}
A_{3}=\frac{-\bar{\delta}_{1}+a_{1} A_{1} r_{1}\left(r_{1}^{2}-\beta^{2}\right) \cosh r_{1}+a_{2} A_{2} r_{2}\left(r_{2}^{2}-\beta^{2}\right) \cosh r_{2}}{\alpha\left(\beta^{2}-\alpha^{2}\right) \cosh \alpha}, \\
A_{4}=\frac{\bar{\delta}_{1}-a_{1} A_{1} r_{1}\left(r_{1}^{2}-\beta^{2}\right) \cosh r_{1}-a_{2} A_{2} r_{2}\left(r_{2}^{2}-\beta^{2}\right) \cosh r_{2}}{\beta\left(\beta^{2}-\alpha^{2}\right) \cosh \beta}, \\
a_{1}=-2 N^{2} /\left(r_{1}^{2}-\beta^{2}\right), \quad a_{2}=-2 N^{2} /\left(r_{2}^{2}-\beta^{2}\right), \\
A_{1}=R_{e} N^{2} M^{2}\left(\sinh r_{2}\right) / 2 d_{1}, \quad A_{2}=-R_{e} N^{2} M^{2}\left(\sinh r_{1}\right) / 2 d_{1}, \\
d_{1}=\left(r_{1}^{3}+\left(T_{1}-T_{2}-\alpha^{2}\right) r_{1}\right) \sinh r_{2} \cosh r_{1} \\
-\left(r_{2}^{3}+\left(T_{1}-T_{2}-\alpha^{2}\right) r_{2}\right) \sinh r_{1} \cosh r_{2}, \\
\bar{\delta}_{1}=R \delta_{1}-2 N^{2}\left(r_{1} A_{1} \cosh r_{1}+r_{2} A_{2} \cosh r_{2}\right), \\
r_{1}=\left(\left(\begin{array}{cc}
\left.\left.-X_{1}+\sqrt{X_{1}^{2}-4 X_{2}}\right) / 2\right)^{1 / 2}, \quad r_{2}=\left(\left(-X_{1}-\sqrt{X_{1}^{2}-4 X_{2}}\right) / 2\right)^{1 / 2}, \\
X_{1}=T_{1}-T_{2}-\left(\alpha^{2}+\beta^{2}\right), \quad X_{2}=\alpha^{2} \beta^{2}-T_{1} \alpha^{2}+T_{2} \beta^{2},
\end{array}\right.\right. \\
T_{1}=N^{4} M^{2} /\left(1-N^{2}\right), \quad T_{2}=N^{2} M^{2} /\left(1-N^{2}\right) \quad \text { and } \quad \beta^{2}=\alpha^{2}-2 i \alpha R_{e} /\left(2+\mu_{1}\right) .
\end{gathered}
$$


The solutions to (3.6)-(3.7) are

$$
\begin{aligned}
\phi_{20}^{(1)}(y) & =G_{1}(y)-2 N^{2} G_{2}(y)-\frac{2 N C_{2}}{M} \cosh (N M y)+\frac{C_{1} y^{2}}{2\left(1-N^{2}\right)}+C_{3}, \\
\xi_{20}(y) & =C_{2} \sinh (N M y)+f(y)-\frac{C_{1} y}{2\left(1-N^{2}\right)},
\end{aligned}
$$

where

$$
\begin{gathered}
C_{1}=\frac{i \alpha R_{e}}{2+\mu_{1}} D_{3}+\frac{\alpha^{2}}{2} D_{1}-\frac{1}{2} D_{4}-N^{2} D_{5} \\
C_{2}=\frac{C_{1}-2\left(1-N^{2}\right)\left(f(1)+0.5 D_{2}\right)}{2\left(1-N^{2}\right) \sinh (N M)} \\
C_{3}=-0.5 D_{1}-G_{1}(1)+2 N^{2} G_{2}(1)+\frac{2 N C_{2}}{M} \cosh (N M)-\frac{1}{2\left(1-N^{2}\right)} C_{1}, \\
D_{1}=\phi_{1}^{*(2)}(1)+\phi_{1}^{(2)}(1), \quad D_{2}=\xi_{1}^{*(1)}(1)+\xi_{1}^{(1)}(1), \quad D_{3}=\phi_{1}^{*(2)}(1)-\phi_{1}^{(2)}(1) \\
D_{4}=\phi_{1}^{*(4)}(1)+\phi_{1}^{(4)}(1), \quad D_{5}=\xi_{1}^{*(2)}(1)+\xi_{1}^{(2)}(1), \\
G_{2}(y)=\int f(y) d y, \quad G_{1}(y)=\frac{-i \alpha R_{e}}{2+\mu_{1}} \int\left(\phi_{1}(y) \phi_{1}^{*(2)}(y)-\phi_{1}^{*}(y) \phi_{1}^{(2)}(y)\right) d y
\end{gathered}
$$

and

$$
\begin{aligned}
f(y)= & -\frac{2 i \alpha R_{e} N^{2} M^{2}}{\left(2+\mu_{1}\right)\left(1-N^{2}\right)}\left[A_{3} A_{4}^{*}\left(\beta^{* 2}-\alpha^{2}\right) F\left(\alpha, \beta^{*}\right)\right. \\
& +A_{3} A_{1}^{*} a_{1}^{*}\left(r_{1}^{* 2}-\alpha^{2}\right) F\left(\alpha, r_{1}^{*}\right)+A_{3} A_{2}^{*} a_{2}^{*}\left(r_{2}^{* 2}-\alpha^{2}\right) F\left(\alpha, r_{2}^{*}\right) \\
& +A_{4} A_{3}^{*}\left(\alpha^{* 2}-\beta^{2}\right) F(\alpha, \beta)+A_{4} A_{4}^{*}\left(\beta^{* 2}-\beta^{2}\right) F\left(\beta, \beta^{*}\right) \\
& +A_{4} A_{1}^{*} a_{1}^{*}\left(r_{1}^{* 2}-\beta^{2}\right) F\left(\beta, r_{1}^{*}\right)+A_{4} A_{2}^{*} a_{2}^{*}\left(r_{2}^{* 2}-\beta^{2}\right) F\left(\beta, r_{2}^{*}\right) \\
& +A_{1} A_{3}^{*} a_{1}\left(\alpha^{2}-r_{1}^{2}\right) F\left(\alpha, r_{1}\right)+A_{4} A_{4}^{*} a_{1}\left(\beta^{* 2}-r_{1}^{2}\right) F\left(r_{1}, \beta^{*}\right) \\
& +A_{1} A_{1}^{*} a_{1} a_{1}^{*}\left(r_{1}^{* 2}-r_{1}^{2}\right) F\left(r_{1}, r_{1}^{*}\right)+A_{1} A_{2}^{*} a_{1} a_{2}^{*}\left(r_{2}^{* 2}-r_{1}^{2}\right) F\left(r_{1}, r_{2}^{*}\right) \\
& +A_{2} A_{3}^{*} a_{2}\left(\alpha^{2}-r_{2}^{2}\right) F\left(\alpha, r_{2}\right)+A_{2} A_{4}^{*} a_{2}\left(\beta^{* 2}-r_{2}^{2}\right) F\left(r_{2}, \beta^{*}\right) \\
& \left.+A_{2} A_{1}^{*} a_{2} a_{1}^{*}\left(r_{1}^{* 2}-r_{2}^{2}\right) F\left(r_{2}, r_{1}^{*}\right)+A_{2} A_{2}^{*} a_{2} a_{2}^{*}\left(r_{2}^{* 2}-r_{2}^{2}\right) F\left(r_{2}, r_{2}^{*}\right)\right], \\
F(A, B)= & \frac{\sinh ((A+B) y)}{(A+B)\left[(A+B)^{2}-N^{2} M^{2}\right]}-\frac{\sinh ((A-B) y)}{(A-B)\left[(A-B)^{2}-N^{2} M^{2}\right]} .
\end{aligned}
$$

\section{References}

[1] T. Ariman, M. A. Turk and N. D. Sylvester, "Microcontinuum fluid mechanics. A review", Int. J. Engng. Sci. 11 (1973) 905-930.

[2] T. Ariman, M. A. Turk and N. D. Sylvester, "Applications of microcontinuum fluid mechanics", Int. J. Engng. Sci. 12 (1974) 273-293. 
[3] G. Böhme and R. Friedrich, "Peristaltic flow of viscoelastic liquids", J. Fluid Mech. 128 (1983) $109-122$.

[4] T. D. Brown and T. K. Hung, "Computational and experimental investigations of two-dimensional nonlinear peristaltic flows", J. Fluid Mech. 83 (1977) 249-272.

[5] J. C. Burns and T. Parkes, "Peristaltic motion", J. Fluid Mech. 29 (1969) 731-743.

[6] A. C. Eringen, "Theory of micropolar fluids", J. Math. Mech. 16 (1966) 1-18.

[7] A. C. Eringen, Microcontinuum field theories. Vol. II: Fluent media (Springer, New York, 2001).

[8] Y. C. Fung and C. S. Yih, "Peristaltic transport", Trans. ASME J. Appl. Mech. 35 (1968) 669-675.

[9] R. Girija Devi and R. Devanathan, "Peristaltic transport of micropolar fluid", Proc. Indian Acad. Sci. 81(A) (1975) 149-163.

[10] M. Y. Jaffrin and A. H. Shapiro, "Peristaltic pumping", Annual Rev. Fluid Mech. 3 (1971) 13-36.

[11] K. A. Kline and S. J. Allen, "Nonsteady flows of fluids with microstructure", Phys. Fluids 13 (1970) 263-270.

[12] T. K. Mittra and S. N. Prasad, "On the influence of wall properties and Poiseuille flow in the peristalsis", J. Biomechanics 6 (1973) 681-693.

[13] D. Philip and Peeyush Chandra, "Peristaltic transport of simple micro fluid", Proc. Nat. Acad. Sci. India 65(A) (1995) 63-74.

[14] J. Prakash and P. Sinha, "Squeeze film theory for micropolar fluids", Tran. ASME J. Lubrication Tech. 98 (1976) 139-144.

[15] G. Radhakrishnamacharya, "Long wave length approximation to peristaltic motion of a power law fluid", Rheol. Acta 21 (1982) 30-35.

[16] K. K. Raju and R. Devanathan, "Peristaltic motion of a non-Newtonian fluid. I", Rheol. Acta 11 (1972) 170-178.

[17] B. V. Rathish Kumar and K. B. Naidu, "A numerical study of peristaltic flows", Compt. Fluids 24 (1995) 161-176.

[18] K. P. Selverov and H. A. Stone, "Peristaltically driven channel flows with applications toward micromixing", Phys. Fluids 13 (2001) 1837-1859.

[19] A. H. Shapiro, M. Y. Jaffrin and S. L. Weinberg, "Peristaltic pumping with long wavelength at low Reynolds number”, J. Fluid Mech. 37 (1969) 799-825.

[20] L. M. Srivastava, "Peristaltic transport of couple-stress fluid", Rheol. Acta 25 (1986) 638-641.

[21] L. M. Srivastava and V. P. Srivastava, "Peristaltic transport of blood: Casson model. II", $J$. Biomechanics 17 (1984) 821-829.

[22] S. Takabatake and K. Ayukawa, "Numerical study of two-dimensional peristaltic flows", J. Fluid Mech. 122 (1982) 439-465.

[23] P. Tong and D. Vawter, "An analysis of peristaltic pumping", Trans. ASME J. Appl. Mech. 39 (1972) 857-862.

[24] F. Yin and Y.C. Fung, "Comparison of theory and experiment in peristaltic transport", J. Fluid Mech. 47 (1971) 93-112. 